\title{
Comparing the influence of 1-day versus 3-day low- residue diet on bowel preparation for colonoscopy:a randomized controlled trial
}

\section{Li Jiao ( $\nabla_{\text {jiaoli1985211@163.com ) }}$}

Hebei Medical University Third Affiliated Hospital https://orcid.org/0000-0001-7888-3526

Junmin Wang

Hebei Medical University Third Affiliated Hospital

\section{Wenjuan Zhao}

Hebei Medical University Third Affiliated Hospital

\section{Huan $\mathrm{Ma}$}

Hebei Medical University Third Affiliated Hospital

\section{Xiao Fan}

Hebei Medical University Third Affiliated Hospital

\section{Liang Yang}

Hebei Medical University Third Affiliated Hospital

\section{Xuejuan Zhu}

Hebei Medical University Third Affiliated Hospital

\section{Research article}

Keywords: colonoscopy, bowel preparation, low-residue diet, duration, bowel-cleansing quality

Posted Date: October 15th, 2019

DOI: https://doi.org/10.21203/rs.2.15706/v1

License: (c) (i) This work is licensed under a Creative Commons Attribution 4.0 International License. Read Full License 


\section{Abstract}

Backgroud :A low-residue diet (LRD) can potentially reduce the hunger of patients while improving compliance and tolerance,without compromising the quality of bowel preparation. This study investigated the effects of 1-day low-residue diets compared with 3-day for colonoscopic bowel preparation .

Methods: Patients undergoing bowel preparation before colonoscopy were randomly divided into 1day and 3-day LRD groups. Oral polyethylene glycol (PEG) electrolyte solution and simethicone were applied to all patients submitted to bowel preparation. The primary outcome measure included the quality of the bowel preparation rated by the Boston bowel preparation scale(BBPS), and the secondary measures included (i) cecal intubation time and withdrawal time, (ii) polyp detection rate, (iii) patient tolerance and (iv) the willingness of the patient to repeat the diet protocol thereafter.

Results: No significant difference was detected in regards the quality of the bowel preparation when comparing the two groups, since the mean of BBPS score was 6.54 versus 6.55 in the 1-and 3-day LRD groups. The cecal intubation times,withdrawal times, polyp detection rates,and the patient tolerance (i.e.hunger-comfort score[1]) showed no significant difference between the 1- and 3-day LRD groups. However,overall satisfaction was higher with the 1-day LRD group than with the 3-day LRD group.

Conclusion: The 1-day and 3-day low-residue diets showed no major effect on bowel preparation previously to colonoscopy, while the tolerance and satisfaction of patients were higher after 1-day lowresidue diet. Trial registration: Clinical Trial Registry Identifier:ChiCTR1900025843.Date of registration:September 10,2019 "Retrospectively registered”. Key words: colonoscopy; bowel preparation; low-residue diet; duration; bowel-cleansing quality

\section{Introduction}

Over the year, the incidence of colorectal cancer has continuously increased, ranking third to fifth most frequent type of cancer among all malignant tumors worldwide [2]. As a long-standing outpatient procedure, colonoscopy is considered the "gold standard" for screening and prevention of colorectal cancer [3]. However, studies have shown that $4-5 \%$ of colon cancers cannot be properly detected by a single colonoscopy [4]. One of the major reasons for undetectable colon cancer is the inadequate bowel preparation [5]. Poor bowel preparation can reduce the detection rate of high-risk adenoma and the completion rate of enteroscopy [6]. Therefore, a good quality of bowel preparation before medical examination is essential.

Patient compliance is required for an adequate bowel preparation. For this, a series of procedures aiming to improve patient compliance have been developed, such as improved colonoscopy prep diets, use of reduced-volume laxatives, and phased administration of polyethylene glycol (PEG) electrolytes.

Nevertheless, bowel preparation before colonoscopy is still uncomfortable and burdensome for a large number of patients, and it is still considered a major obstacle for colon tumor screening in high-risk populations [7]. 
Dietary improvements for colonoscopy purposes have been consistently pursued [8]. One common practice to improve the quality of bowel preparation has been the use of clear liquid diets one day before colonoscopy. Still, this dietary approach has been reported to increase hunger of treated patients and to reduce patient compliance[9]. Alternatively, other studies have shown that low-residue diet (LRD) can reduce hunger in patients and improve compliance and tolerance without affecting the quality of bowel preparation [8-10]. However, the number of reports describing the potential impact of LRD duration on preprocedure bowel preparation is still limited.

In this study, we compared the effects of LRD (one and three-day treatments) to PEG electrolyte solution and simethicone in regards the quality of bowel preparation. For this, we initially evaluated the quality of bowel preparation, and the assessed secondary outcome measures which included patient tolerance, satisfaction, polyp detection rate and endoscopic intubation and withdrawal time.

\section{Material And Methods}

\section{Study design}

This study was a randomized, controlled, single-blind, prospective trial comparing two dietary regimens prior to colonoscopy: a 1-day low-residue diet(LRD) vs. a 3-day LRD the day before colonoscopy. The study protocol was approved by the Ethics Committee of the third Hospital of Hebei Medical University, and was registered at Chinese Clinical Trial Registry(ChiCTR1900025843).

\section{Subjects:}

A cohort of patients who underwent colonoscopy from April to August 2019 at the Third Hospital of Hebei Medical University,in Shijiazhuang city,Hebei province was selected for our work. All recruited patients signed an appropriate informed consent to be allowed to participate in this study. Both experimental protocol and informed consent were submitted to the Ethics Committee Formal enrollment and study were conducted solely upon approval.The following inclusion criteria were utilized: (i) patients' age should be between 18 and 80 years old, (ii) patients should be ready for colonoscopy, and (iii) patients should be undergoing colorectal cancer screening or having non-specific gastrointestinal symptoms. The following exclusion criteria were utilized: (i) patients who were unable to cooperate with the examination; (ii) patients who underwent gastrointestinal surgery; (iii) patients with severe diseases in heart, brain, kidney, liver and other organs; (iv) patients who could not tolerate the colonoscopy procedure; (v) patients with contraindications to colonoscopy, including bowel obstruction or perforation, severe intestinal infection or toxic megacolon, consciousness disorder and unstable vital signs; (vi) patients whose colonoscopy failed to reach ileocecum due to reasons other than bowel preparation (such as intestinal stricture caused by tumor); (vii) pregnant and lactating patients; (viii) patients who suffered a stroke or dementia; (ix) patients with anxiety or depressive psychosis; and (x) PEG allergic patients.A total of 240 patients were recruited. Upon signing an informed consent, eligible patients were randomly divided into two groups, according to the period of supplementation with a low-residue diet (one or three-day treatment).Each subject obtained a sealed opaque envelope whose contents were determined by a 
random number table prepared by an independent researcher. Patients were allowed to eat rice porridge, noodles, steamed bread, bread, tofu, egg soup, chicken, some peeled, seeded fruits and cooked vegetablesbut devoid of untreated vegetables, fruits and coarse grains.

\section{Bowel preparation schedules:}

Patients were instructed to adopt a low residue diet one day or three days before colonoscopy, according to their respective grouping, and then fasted for about 11 hours $(10 \mathrm{pm}$ to $9 \mathrm{am}$ on the day of the procedure).Patients were not allowed to discuss grouping in front of the enteroscopy operators. Eventual issues were directly treated with nursing personnel. Control patients were administered with three liters of PEG electrolyte solution betwenn 9-11am on the day of colonoscopy, followed by oral administration of $30 \mathrm{ml}$ simethicone. Colonoscopy was performed between 3-5pm on the same day.Endoscopic diagnosis and treatment were conducted using an Olympus $\mathrm{CF} \mathrm{H-290} \mathrm{colonoscope.} \mathrm{Endoscopists} \mathrm{were} \mathrm{unaware} \mathrm{of}$ the patient grouping.Colonoscopy experience in at least 1,000 cases was used as a threshold to select the professionals involved in this procedure.

\section{Outcome indicators:}

The following measures were taken in consideration for our dietary evaluation:

(I) Patient profiling (age, gender, weight, education level, constipation history, colonoscopy history, and abdominal symptoms);

(II) Colonoscopy profiling (percentage of arrival at the cecum, intubation time, withdrawal time, and the detection rate of colon polyps).

(III) Bowel-cleansing quality score: assessed according to Boston bowel preparation scale (BBPS) [11]. For this, the BBPS divides the colon into three regions: the right side (cecum and ascending colon), the transverse section (including the hepatic flexure and splenic flexure), and the left side (descending colon, sigmoid colon, and rectum). A 4-point classification scale was utilized as previously reported [12]. The three colon regions were scored accordingly, and the quality of bowel preparation was assessed by its total score (i.e. 4-point scale multiplied by 3 ). These total scores were mainly divided into 5 grades: excellent (8-9), good (6-7), medium (4-5), poor (2-3) and extremely poor (0-1). Grades over 4 (medium) were considered as qualified for further analyses [12].

(IV) Patient tolerance and satisfaction: patients were submitted to a questionnaire, before the examination, on the day of colonoscopy. According to the digital analogue scale method, the research group designed a hunger-comfort scale [1], which evaluated the hunger-comfort condition of the patients, where " 1 " indicated comfort and " 10 " indicated hunger. The diet acceptance of the patients before colonoscopy was graded in 5 distinct levels: very simple acceptance, simple acceptance, tolerable, unacceptable, and difficult to accept [1]. The willingness to repeat the diet protocol for bowel preparation was evaluated accordingly [13]. Eventual occurrence of adverse reactions, such as nausea, vomiting, abdominal distension and discomfort, was carefully annotated. 


\section{Sample size calculation}

The primary focus of this study was to evaluate the quality of bowel preparation by BBPS scoring (ranging from 0 to 9). Our previous studies in healthy and with abdominal symptoms polulation have showed that related values are well spread across the 10-point with a mean of 6.5 points, and standard deviation of 1.6 points. We considered a 1-point average difference between groups minimally clinically important. With an s.d. of 1.6 and 42 subjects per arm, the independent $t$-test would provide $80 \%$ power to detect a between group difference of 1 point at a two-sided a of 0.05 . Assuming a dropout rate of $20 \%$ ,we thus aimed at enrolling 51 subjects in each arm to achieve an effective sample size of at least 42 per group.

6. Statistical analysis:

SPSS 19.0 software (SPSS Inc., Chicago, Illinois, USA) was used for our statistical data analysis. Data measurement was represented by mean plus/minus standard deviation. Homogeneity of variance was analyzed and, if the variance was equal, a t-test was applied for pairwise comparison between independent samples. If variance was unequal, Kruskal Wallis test was used, and the difference was statistically significant when $P<0.05$. $\chi^{\square}$ test was used for data counting, and statistical significance was set at $\mathrm{P}<0.05$.

\section{Results}

\section{Clinical data}

A total of 240 outpatients and inpatients were initially enrolled in this study. Among them, 9 patients in the 1-day LRD group and 10 patients in the 3-day LRD group were removed from this study due to (i) failure of attending colonoscopy, (ii) non-adherence to dietary plans, and/or (iii) decline of participation. Therefore, a total of 221 patients (1-day LRD group, $n=111$; 3-day LRD group, $n=110$ ) were included in this study(Fig. 1). As shown in Table 1, no significant difference was observed when comparing the baseline clinical data between the two groups $(P>0.05)$.

\section{Quality of bowel preparation}

The quality of colon preparation (whole organ and individual parts) was evaluated using the BBPS score. As shown in Table 2, in the 1-day LRD group, the scores of the left side, the transverse, and the right side of the colon were $2.34 \pm 0.56,1.99 \pm 0.58$, and $2.21 \pm 0.59$, respectively, and the total score was $6.54 \pm 1.45$. In the 3-day LRD group, the scores of the left side, the transverse, and the right side of the colon were 2.21 $\pm 0.66,2.13 \pm 0.74$, and $2.21 \pm 0.66$, respectively, and the total score was $6.55 \pm 1.86$. Therefore, no significant difference was detected between these two groups $(P>0.05)$. The number of cases with qualified bowel preparation (total BBPS score $\geqq 4$ ) in the 1-day group (107 cases, $96.4 \%$ ) was higher than that in 3-day group (100 cases, $91 \%)$. 


\section{Colonoscopy}

As shown in Table 3, the average intubation time was $10.07 \pm 2.46 \mathrm{~min}$ in the 1-day LRD group, and 10.36 $\pm 2.41 \mathrm{~min}$ in the 3-day LRD group. The average withdrawal time was $9.77 \pm 2.45 \mathrm{~min}$ in the 1 -day LRD group, and $10.16 \pm 1.76 \mathrm{~min}$ in the 3-day LRD group. The polyp detection rate was $19 \%$ in the 1-day LRD group and $17.3 \%$ in the 3-day LRD group. No significant different between the two groups was observed for any of these indicators. In both groups, colonoscope was successfully inserted and colonoscopy was completed accordingly.

4. Comparison of patient tolerance and adverse reactions

The hunger-comfort scale was $2.71 \pm 1.91$ in the 1-day LRD group, and $2.88 \pm 2.01$ in the 3-day LRD group, indicating no significant difference between these groups $(P>0.05)$. The proportion of very easy compliance and easy compliance among the patients supplemented with the 1-and 3-day LRD was $72.07 \%$ and $39.09 \%$, respectivelysignificantly different $(P<0.05)$, indicating that the 1 -day LRD was the preferred condition for the patients. Nevertheless,

\section{Discussion}

An adequate bowel preparation is largely dependent on effective colon cleansing, supplements and dietary restrictions [14]. A number of studies have currently focused on the effectiveness, safety, dosage and onset of action of laxatives [15-17].However, there are few studies on the effectiveness and patient tolerance of pre-colonoscopic diet for bowel preparation. Particularly, a few reports have compared the effects of clear liquid diet $\mathbb{C L D}$ \and low-residue diet (LRD) in the pre-colonoscopy bowel preparation. LRD on the day before colonoscopy seems to be as effective as CLD for quality of bowel preparation, but it enables a higher patient tolerabilityand satisfaction $[18,19]$. Accordingly, the European Society of Gastrointestinal Endoscopy (ESGE) and the American Society for Gastrointestinal Endoscopy (ASGE) have well recommended LRD before colonoscopy [17] [20]. Nevertheless, the differential effects (if any) due to proper timing of LRD administration, before colonoscopy, required further investigations. Some scholars recommend an LRD for 3 days prior to colonoscopy bcause of the slow transit time in some patients, while some scholars recommended an LRD for 1 day. ESGE guidelines recommend, based on experience, 1-day LRD for bowel preparation, but without any strong data from evidence-based studies. Therefore, here we performed a randomized, controlled and single-blind trial study which confirmed that the quality of bowel preparation quality after 1-day and 3-day LRD before colonoscopy was basically equivalent. Moreover, our current study has confirmed that the polyp detection rate is similar in patients submitted to 1- or 3-day LRD. These results are consistent with a very recent report which shows no 
significant difference in the quality of bowel preparation in similar LRD treated groups $(n=390)$ [21]. In this study, the proportion with qualified bowel preparation (total BBPS score $\geq 4$ ) was more than $91 \%$ in both groups, indicating that LRD was sufficient to ensure adequate bowel preparation.

By comparing both LRD groups in regards the tolerance, satisfaction and willingness to repeat the preprocedure diet, it was observed that the subjects preferred the 1-day LRD due to the shorter time of diet and easier compliance. However, the comfort-hunger scale showed no significant difference in comfort between these two groups. Similarly, by comparing the acceptance of the diet protocol between the 1-and 3-day LRD groups, it was found that the proportion of subjects with very easy and easy compliance in the 1-day LRD group was significantly higher than that in the 3-day group. Moreover, the patients from the 1day LRD group were more willing to repeat the diet for bowel preparation than those in the 3-day LRD group. These results are consistent with the current literature [21].

The percentage of arrival at the cecum, intubation time and withdrawal time between the two groups were further compared, and no significant difference was observed. Of note, it was found that the mean insertion and withdrawal time in this study were 2 mins, which were longer than the usual. In fact, LRD could ensure adequate bowel preparation and clarity of the mucous membrane which, in this case, could increase the polyp detection rate and, consequently, lead to extra time consumption to perform endoscopic removal of colorectal polyps. Another possibility is that the colonoscopy operators may have paid more attention to the identification of mucous lesions, increasing the operation time of colonoscopy.

In our study, there was no obvious restriction on the types of LRD used, so the this diet had a larger variety of options and was more practical. The avoidance of pre-packaged foods [22] and specified low residue foods increased the compliance and acceptance by the patients. However, the calories and amount of food consumed were disregarded, and more detailed analysis is warranted. In addition, the interval between the last laxative administration and the beginning of colonoscopy was within 6 hours, which ensured the high quality of bowel preparation in this study. This procedure was consistent with other studies previously conducted [23]. Previous research has put forward the concept of "time interval" in colonic cleansing, that is, the colonoscopy should be performed within 4 to 6 hours after bowel preparation where, after this time interval, the contents of small intestine would continue to enter the colon, thus affecting the colonic-cleansing quality [24].

So far, no consensus regarding PEG dosage has been established. Various studies have indicated that the effect of split-dose and one-time administration of PEG may be equivalent but, apparently, the splitdose regime can increase the tolerance and comfort of patients $[25,26]$. In contrast, split-dose administration of PEG may also cause nocturnal diarrhea and affect the quality of sleep. In the present study, colonoscopy was performed in all the patients during afternoon, and three liters of PEG was administrated at 10 am on the same day, which effectively avoided this issue.

Despite the consistency and clinical value of our study, further clarifications are still warranted. For instance, the impact of other diseases that may negativelyaffect bowel preparation quality, such as diabetes and chronic constipation, were not considered, [27]. In addition, detailed medication history was 
not included in our questionnaire, and the administration of drugs that cause constipation, such as opioids and tricyclic antidepressants, could lead to biased results [28]. However, since this study is a random trial, we believe that these limitations do not affect the results of bowel preparation. In contrast, since this study is also a single-center trialand the sample size was relatively small, our results deserve further validation with a larger scale multicenter trial.

\section{Conclusions}

In conclusion, our work confirmed that the quality of bowel preparation after 1-day and the 3-day LRD diet is equivalent. Still, a 1-day LRD appears to increase the comfort of bowel preparation and it is more acceptable to the subjects. These findings reduce the probability of poor bowel preparation, caused by diet before colonoscopy, and the probability of repeated colonoscopy, which certainly impacts on the costs of early diagnostics and the screening of colorectal cancers.

\section{Declarations}

\section{Acknowledgements}

None

\section{Funding}

None

\section{Availability of data and materials}

The datasets generated during the current study are not publicly available due privacy but are available from the corresponding author on reasonable request.

\section{Abbreviations}

PEG polyethylene glycol

BBPS Boston bowel preparation scale

LRD low-residue diet

CLD clear liquid diet

ESGE European Society of Gastrointestinal Endoscopy

ASGE American Society for Gastrointestinal Endoscopy

\section{Authors' contributions}


$\mathrm{JL}$, made substantial contributions to conception and design,or acquisition of data,or analysis and interpretation of data;been involved in drafting the manuscript and revising it critically for important intellectual content.WJM,made substantial contributions to conception and design;been involved in drafting the manuscript and revising it critically for important intellectual.ZWJ,collected and assembled the data; provided substantive suggestions for revision and critically reviewed subsequent iterations the manuscript.MH,collected and assembled the data; provided substantive suggestions for revision and critically reviewed subsequent iterations the manuscript; Reviewed and approved final version of the paper; Responsible for ensuring that questions related to the accuracy or integrity of any part of the work were appropriately investigated and resolved.FX,YL and ZXJ Collected and assembled the data; Interpreted the results; provided substantive suggestions for revision and critically reviewed subsequent iterations the manuscript; Reviewed and approved final version of the paper; Responsible for ensuring that questions related to the accuracy or integrity of any part of the work were appropriately investigated and resolved.All authors have read and approved the manuscript.

\section{Notes}

\section{Ethics approval and consent to participate}

This study was conducted in accordance with the guidelines of the Declaration of Helsinki .The study protocol was approved by the Ethics Committee of the Third Hospital of Hebei Medical University with approval code 2019-025-11. Written informed consent according to standards of the local ethics committees was obtained from all patients.

\section{Consent for publication}

Not applicable.

Competing interests

The authors declare that they have no competing interests.

\section{Consent for publication}

Not applicable.

\section{Publisher's Note}

Springer Nature remains neutral with regard to jurisdictional claims in published maps and institutional affiliations.

\section{Contributor Information}


${ }^{1-7}$ Department of Gastroenterology, the Third Hospital ,Hebei Medical University,Shijiazhuang, China.

\section{References}

1. Andrea Melicharkova, Jennifer Flemming, Stephen Vanner et al(2013). A Low-Residue Breakfast Improves Patient Tolerance Without Impacting Quality of Low-Volume Colon Cleansing Prior to Colonoscopy:A Randomized Trial. Am J Gastroenterol,108:1551-1555; doi:10.1038/ajg.2013.21.

2. Chen $\mathrm{W}$,Zheng R,Zeng $\mathrm{H}$, et al(2015).Annual report on status of cancer in China[J].Chin J Cancer Res,27(1):2-12.DOI:10.3978/j.issn.1000-9604.2015.01.06.

3. Li Junqiang, Zhao Zhongye, Yu Enda(2014). Application Status of Intestinal Preparation Quality Assessment Scale[J]. Chin J Dig Endosc,31ष9):539-542.

4. Haseman JH, Lemmel GT, Rahmani EY, Rex DK(1997). Failure of colonoscopy to detect colorectal cancer: evaluation of 47 cases in 20 hospitals. Gastrointest Endosc , 45:451-455.

5. Faiss S(2011).The missed colorectal cancer problem. Dig Dis,29 Suppl 1:60-63.

6. Harewood GC, Sharma VK, de Garmo P(2003).Impact of colonoscopy preparation quality on detection of suspected colonic neoplasia. Gastrointest Endosc ,58:76-79.

7. Harewood GC, Wiersema MJ, Melton LJ III(2002). A prospective, controlled assessment of factors influencing acceptance of screening colonoscopy. Am J Gastroenterol,97:3186-94.

8. Soweid AM, Kobelssy AA, Jamali FR, et al(2010). A randomized single-blind trial of standard diet versus fiber-free diet with polyethylene glycol electrolyte solution for colonoscopy preparation.Endoscopy,42:633-638.

9. David R. Stolpman, Craig A. Solem, Dianna Eastlick, et al(2014).A Randomized Controlled Trial Comparing a Low-Residue Diet Versus Clear Liquids for Colonoscopy Preparation. J Clin Gastroenterol,48:851-55.

10. Douglas L. Nguyen, M. Mazen Jamal, Emily T. Nguyen,et al(2016).Low-residue versus clear liquid diet before colonoscopy: a meta-analysis of randomized, controlled trials. Gastrointestinal endoscopy,48:499-507.

11. Gao Yan, Zhang Houde, Lin Muxian, et al(2012).Reliability evaluation of intestinal preparation scale[J]. Chin J Dig Endosc,29ه2ख:78-80.

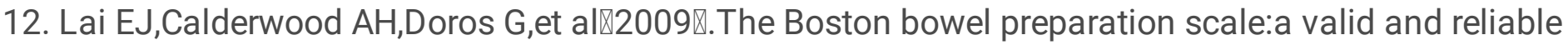
instrument for colonoscopy-oriented research[J].Gastrointest Endose.,69(3 Pt 2):620625.DOI:10.1016/j.gie.2008.05.057.

13. Yoon Suk Jung, Hyo Sun Seok, Dong II Park et al $₫ 2013 \rrbracket$. A Clear Liquid Diet Is Not Mandatory for Polyethylene Glycol-Based Bowel Preparation for Afternoon Colonoscopy in Healthy Outpatients. Gut and Liver,7:681-87.

14. Andrea Melicharkova, Jennifer Flemming, Stephen Vanner, et al(2013).A Low-Residue Breakfast Improves Patient Tolerance Without Impacting Quality of Low Volume Colon Cleansing Prior to Colonoscopy: A Randomized Trial[J]. Am J Gastroenterol,108:1551-55. 
15. Marmo R, Rotondano G , Riccio G et al(2010).Effective bowel cleansing before colonoscopy: a randomized study of split-dosage versus non-split dosage regimens of high-volume versus lowvolume polyethylene glycol solutions .Gastrointest Endosc ,72:313-20.

16. Xie Q, Chen L, Zhao F, et al(2014). A meta-analysis of randomized controlled trials of low-volume polyethylene glycol plus ascorbic acid versus standard-volume polyethylene glycol solution as bowel preparations for colonoscopy.

17. PLOS ONE,9:e99092.

18. Hassan C , East J , Radaelli F et al(2019). Bowel preparation for colonoscopy: European Society of Gastrointestinal Endoscopy (ESGE) guideline-update 2019. Endoscopy,51(8):775-794. doi: 10.1055/a-0959-0505.

19. Douglas L. Nguyen, M. Mazen Jamal, Emily T. Nguyen et al(2016). Low-residue versus clear liquid diet before colonoscopy:a meta-analysis of randomized, controlled trials. Gastrointestinal Endoscopy, 83:499-507.

20. Stolpman DR, Solem CA, Eastlick D, Adlis S, Shaw MJ(2014). A randomized controlled trial comparing a low-residue diet versus clear liquids for colonoscopy preparation: impact on tolerance, procedure time, and adenoma detection rate. J Clin Gastroenterol 2014,48: 851-5.

21. Bowel preparation before colonoscopy: the American Society for Gastrointestinal Endoscopy(ASGE) guideline-2015. Gastrointestinal Endoscopy 2015.

22. Antonio Z. Gimeno-García, Raquel de la Barreda Heuser, Cristina Reygosa(2019).Impact of a 1-day versus 3-day low-residue diet on bowel cleansing quality before colonoscopy: a randomized controlled trial.Endoscopy.

23. Matsumura, M. Arai, H. Ishigami et al(2015). A randomized controlled trial comparing a prepackaged low-residue diet with a restricted diet for colonoscopy preparation: the impact on the results of colonoscopy in adenoma detection. Colorectal Disease,18:37-42.

24. Siddiqui AA,Yang K,Spechler SJ,et al(2019).Duration of the interval between the completion of bowel preparation and the start of colonoscopy predicts bowel- preparation quality[J].Gastrointest Endosc,69ه3Pt2):700-706.DOI:10.1016/

25. gie.2008.09.047.

26. James M. Church(1998). Effectiveness of Polyethylene Glycol Antegrade Gut Lavage Bowel Preparation for Colonoscopy-Timing is the Key! Dis Colon Rectum,41:1223-1225.

27. Gabriel Perreaulta \Adam Goodmanb囚Sebastian Larionc et al(2018). Split-versus single-dose preparation tolerability in a multiethnic population: decreased side effects but greater social barriers. Annals of Gastroenterology,31:356-364.

28. Wu Dong, Li Ji, Yang Hong, et al(2016). Study on the value of taking polyethylene glycol in combination with simethicone overnight to improve the quality of morning colonoscopy. Chin J Dig Endosc,33:751-755.

29. Taylor C, Schubert ML(2001). Decreased efficacy of polyethylene glycol lavage solution in the preparation of diabetic patients for outpatient colonoscopy: a prospective and blinded study. Am J 
Gastroenterology ,96:710-714.

30. Hale WE, Perkins LL, May FE et al(1986).Symptom prevalence in the elderly. An evaluation of age, sex, disease, and medication use. J Am Geriatr Soc,34:333-34

\section{Tables}

Table 1. Comparison of clinical data between patient groups

\begin{tabular}{cccc}
\hline Feature & 1-day LRD group (n=111) & 3-day LRD group (n=110) & $P$-value \\
\hline Age & $47.80 \pm 15.46$ & $46.64 \pm 15.09$ & 0.571 \\
Weight & $61.01 \pm 10.75$ & $61.90 \pm 9.9$ & 0.527 \\
Gender (male) & $39(35.14)$ & $40(36.36)$ & 0.849 \\
Education level (high school and above) & $68(61.26)$ & $61(55.45)$ & 0.162 \\
Constipation history & $20(18.02)$ & $24(21.82)$ & 0.479 \\
Digestive tract symptoms & $81(72.97)$ & $91(82.73)$ & 0.081 \\
Colonoscopy history & $14(12.61)$ & $11(10)$ & 0.540 \\
\hline
\end{tabular}

These results indicate no significant difference in age, weight, gender, education level, constipation, abdominal symptoms and colonoscopy history between the two analyzed groups. Digestive tract symptoms included abdominal pain and/or distension, altered defecation habits, and bloody stool.

Table 2 Boston bowel preparation scale

\begin{tabular}{cccc}
\hline Scaled region & 1-day LRD group $(\mathrm{n}=111)$ & 3-day LRD group $(\mathrm{n}=110)$ & $P$-value \\
\hline Left side of colon & $2.34 \pm 0.56$ & $2.21 \pm 0.66$ & 0.109 \\
Right side of colon & $2.21 \pm 0.59$ & $2.21 \pm 0.66$ & 0.982 \\
Transverse colon & $1.99 \pm 0.58$ & $2.13 \pm 0.74$ & 0.130 \\
Total & $6.54 \pm 1.45$ & $6.55 \pm 1.86$ & 0.983 \\
\hline
\end{tabular}

Results indicate no significant difference in the BBPS scale when comparing the two respective groups.

Table 3 Inter-group comparison of colonoscopy indicators

\begin{tabular}{cccc}
\hline Colonoscopy indicator & 1-day LRD group (N=111) & 3-day LRD group (N=110) & $P$-value \\
\hline Intubation time (min) & $10.07 \pm 2.46$ & $10.36 \pm 2.41$ & 0.375 \\
Withdrawal time (min) & $9.77 \pm 2.45$ & $10.16 \pm 1.76$ & 0.177 \\
Polyp detection rate (\%) & $21(19)$ & $19(17.3)$ & 0.751 \\
\hline
\end{tabular}


Results indicate no significant difference in intubation time, withdrawal time and polyp detection rate between the two respective groups.

Table 4 Inter-group comparison of patient tolerance and adverse reactions

\begin{tabular}{cccc}
\hline Tolerance and adverse reaction & 1-day LRD group & 3-day LRD group & $\begin{array}{c}\text { P- } \\
\text { value }\end{array}$ \\
\hline Hunger-comfort scale & (N=111) & $2.88 \pm 2.01$ & 0.520 \\
Very easy compliance and easy compliance (\%) & $80(72.07)$ & $43(39.09)$ & $<0.001$ \\
Willing to repeat the diet protocol for bowel & $106(95.50)$ & $96(87.27)$ & 0.029 \\
preparation (\%) & & $30(27.27)$ & 0.847 \\
Adverse reaction (\%) & $29(26.12)$ & & 31.029 \\
\hline
\end{tabular}

Results show no significant difference in hunger-comfort scale between the two groups, while the percentage of very easy compliance and easy compliance, and the willingness to repeat the diet protocol for bowel preparation were significantly different. The incidence of adverse reactions was similar between the groups.

\section{Figures}




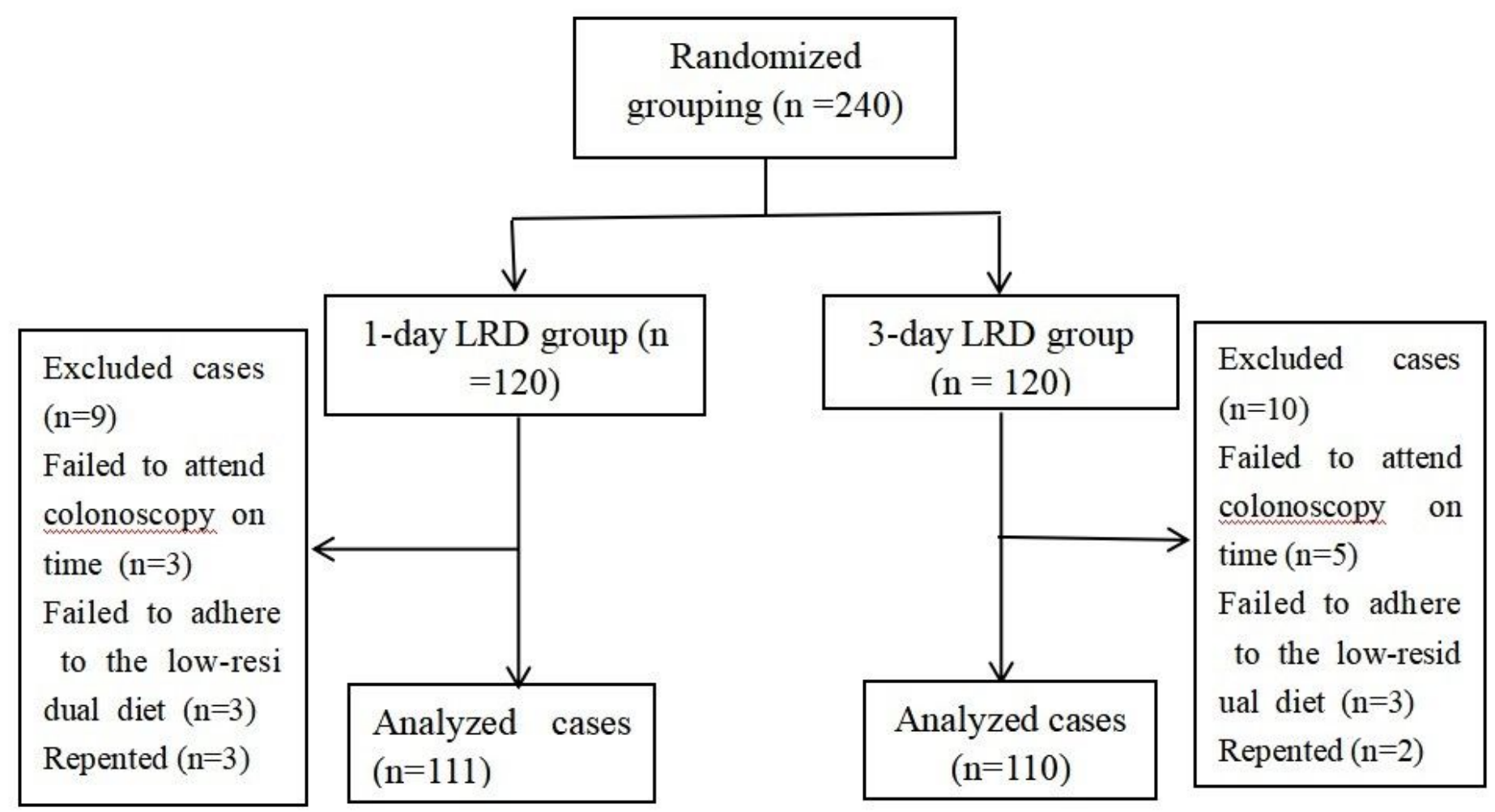

Figure 1. Study process

(flow chart)

Figure 1

Study process 